\title{
Hypersensitivity Reactions and Acute Hepatitis Induced by Therapeutic Doses of Ibuprofen
}

\author{
Maaroufi N*, Othmani S, Ouaz S and Lakhal J \\ Emergency Department, University Tunis ElManar, Tunisia
}

*Corresponding author: Neila Maaroufi, Emergency Department, Jendouba Regional Hospital, University Tunis ElManar, Tunisia, Email: maaroufineila@yahoo.fr

\section{Case Report}

Volume 3 Issue 4

Received Date: November 17, 2018

Published Date: November 30, 2018

DOI: $10.23880 /$ act- 16000142

\section{Abstract}

Drug Reaction with Eosinophilia and Systemic Symptom (DRESS) is a severe adverse drug-induced reaction. Diagnosing DRESS is specifically complicated due to the multiplicity of organs involved. The main culprit drugs are carbamazepine and allopurinol, even though almost 50 drugs have been described to have caused this syndrome. We describe a case of a 34-year old woman admitted to our hospital with acute diffuse, pruritic rash associated with cramp abdominal pain, and fever seven days after starting non-steroidal anti-inflammatory drugs (ibuprofen). Investigation revealed leukocytosis, eosinophilia, and elevated serum transaminases. The diagnosis of DREES syndrome was made, ibuprofen was stopped, and systemic corticotherapy was prescribed with gradually improvement of the rash and symptoms resolution. The patient was discharged home after three weeks of hospitalization.

Keywords: Hypersensitivity Réactions; DRESS syndrome; Ibuprofen; Acute Hepatitis

\section{Introduction}

Drug rash with eosinophilia and systemic symptoms (DRESS) is a complex syndrome with a broad-spectrum of clinical features. However, use of the term DRESS has been inconsistent, because eosinophilia is not a constant clinical finding, Cutaneous and systemic signs are variable [1]. The estimated incidence of this syndrome ranges from 1 in 1000 to 1 in 10,000 drug exposures, adults are more affected than children [2,3]. The main culprit drugs are carbamazepine and allopurinol, even though 50 drugs can induce DRESS [2].

DRESS syndrome including a severe skin eruption, fever, hematologic abnormalities (eosinophilia or atypical lymphocytes), and internal organ involvement, usually 26 weeks after the initiation of drug therapy, with a possibility of persistence or aggravation of symptoms despite the discontinuation of the culprit drug. Given the potential morbidity, wide differential diagnosis, and relatively simple treatment, it is important for emergency physicians to consider this entity in patients with severe rashes.

\section{Patient and observation}

A 34-year old woman was admitted in the emergency department for generalized pruritic erythematous oedematous patches, periorbital oedema (Figure 1) fever and chills. One week before, she had an episode of mild headache, myalgias, arthralgias and dysthermia, therefore, she was treated with ibuprofen reaching atotal take $1200 \mathrm{mg} /$ day. 


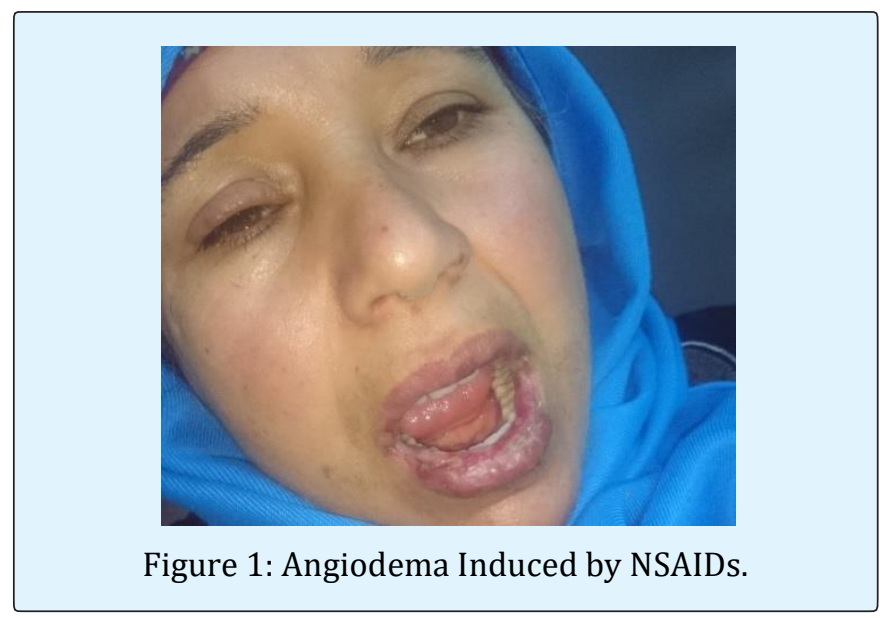

There was no history of herbal Product intake, neither fungi or mushrooms or spoiled food. She had not travelled outside Tunisia in the recent past. She denied any sexual relationship nor realization of tattoos, piercings or any other risky behaviour. On admission patient's physical examination was remarkable for a fever $38,5^{\circ}$, pulse rate was 120 beats per minute, blood pressure was $125 / 62$ $\mathrm{mm} \mathrm{Hg}$, respiratory rate was 22 breaths/ $\mathrm{min}$, and pulseoximetry was $98 \%$ on room air.

Her skin had disseminated dermatosis with papules (figure 2); there were also some pustules on the dorsal area, upper and lower limbs (figure 3). There was no evidence of lymphadenopaty or hepatosplenomegaly. She exhibited no meningismus and had a normal heart, lung, and abdominal examination.

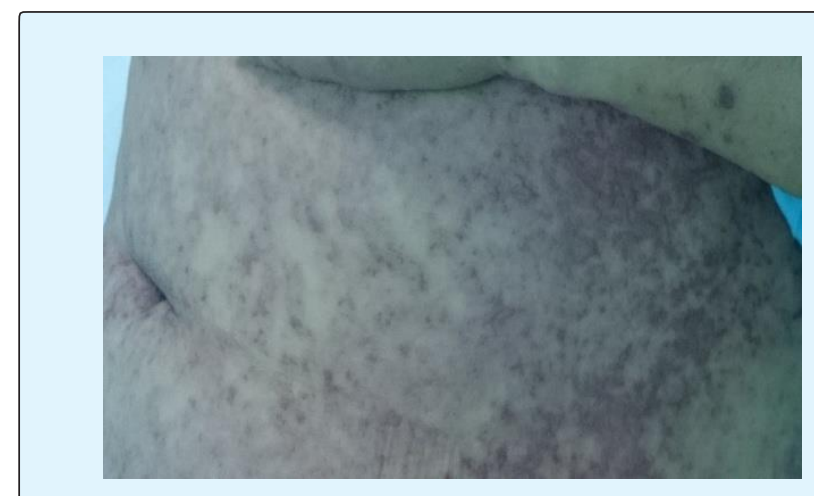

Figure 2: Disseminated Dermatosis with Papules.

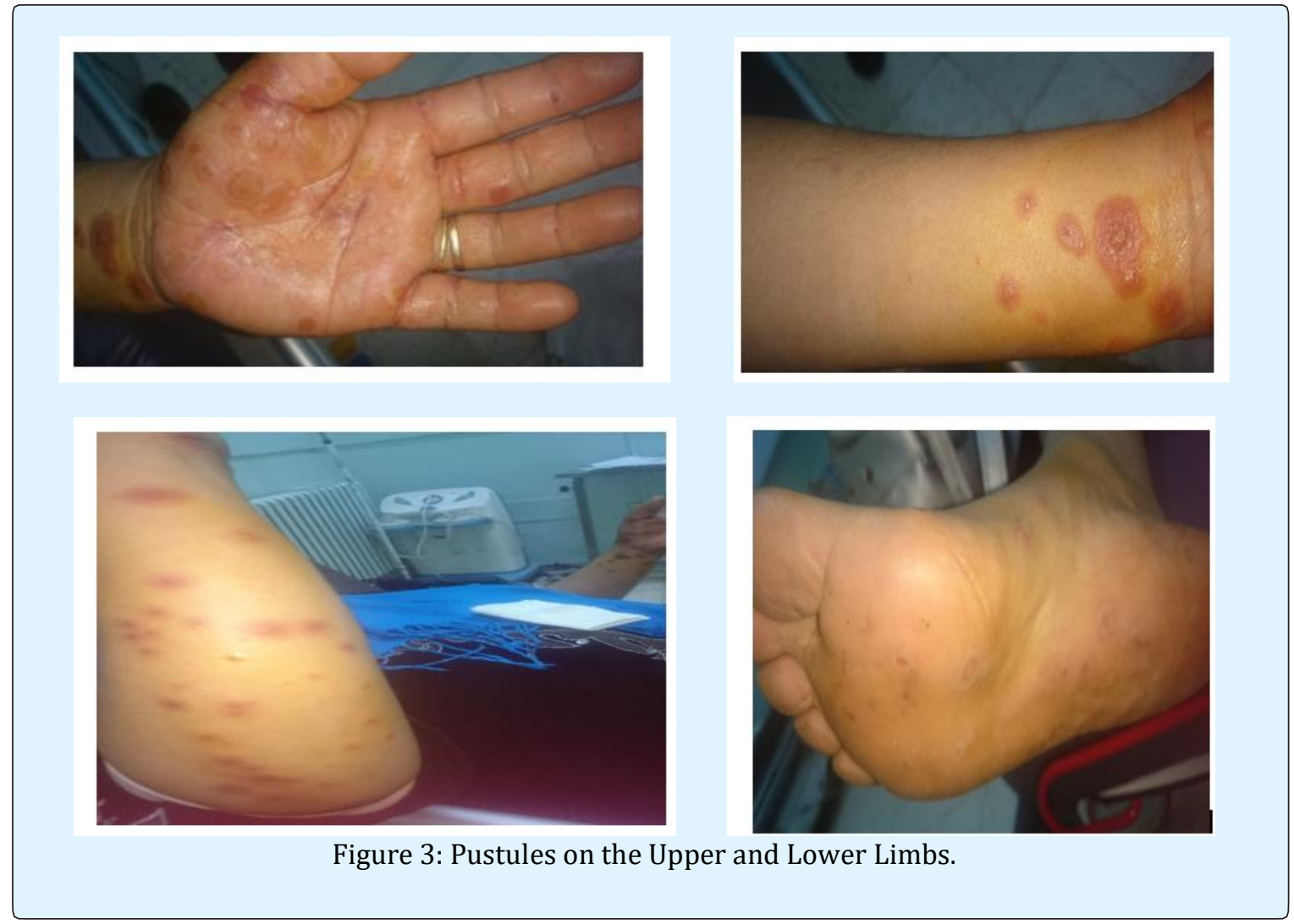


Laboratory examination showed leukocytosis, white blood cell count $15.000 / \mathrm{mm}^{3}$ (normal range 4.000 $10.000 / \mathrm{mm}^{3}$ ), with absolute eosinophil count of 2300 cell/_L, with no atypical lymphocytes. She had also abnormal liver function tests, aspartate aminotransferase (AST) $173 \mathrm{U} / \mathrm{L}$ (10-35 U/L), alanine aminotransferase (ALT) $94 \mathrm{U} / \mathrm{L}(10-40 \mathrm{U} / \mathrm{L})$, and an elevated lactate dehydrogenase level 1123U/L (270-530 U/L). Her Creactive protein was $58 \mathrm{mg} / \mathrm{L}(<10 \mathrm{mg} / \mathrm{L})$. Blood culture and bacteriological tests were negative. Serologic tests for acute infection by hepatotropic virus-hepatitis A, B, C, E, Epstein-Barr virus, Citomegalo virus, human herpes virus were negative.

Chest radiography showed no pathological changes. An abdominal ultrasound was made, revealing no pathological signs. The patient was admitted to the emergency department. The diagnosis of DRESS syndrome was made [using RegiS-CAR criteria, the patient had 4 characteristics-acute skin rash, fever $\left(>38^{\circ} \mathrm{C}\right)$, involvement of liver, and eosinophils above the laboratory limits)]. Non-steroidal anti-inflammatory was stopped, and the patient was treated by oral antihistamines and corticosteroids, prednisolone 60 $\mathrm{mg} /$ day (1 mg/kg/day) with a progressive improvement in the rash. The patient's skin symptoms and laboratory abnormalities started improving on the tenth day of hospitalization. He returned at home after three weeks. One week after discharge, patient symptoms resolved and the laboratory values returned to normal.

\section{Discussion}

Non-steroidal anti-inflammatory drugs NSAIDs are the most important group of drugs involved in hypersensitivity drug reactions [4], and include heterogeneous compounds with very different chemical structures. These reactions can be IgE dependent (immediate reactions), $\mathrm{T}$ cell-mediated (nonimmediate), or induced by a non-specific immunological mechanism related with the blocking of the COX-1 enzyme and the shunting to the lipooxygenase pathway (cross-intolerant reactions) [4]. Cutaneous symptoms are the most frequent, with ibuprofen, naproxen and diclofenac being common culprit drugs worldwide, although others can be involved because patterns of consumption and exposure rates vary between countries [5-7].

A very important proportion of immunological reactions are immediate, with urticaria and anaphylaxis being the typical clinical manifestations. Non-immediate reactions comprise a number of heterogeneous entities ranging from mild exanthema to severe DRESS syndrome, as well as organ-specific reactions such as hepatitis or pneumonitis. Cross-intolerant reactions appear to nonchemically related drugs, and involve respiratory airways, skin or both.

DRESS syndrome was first introduced in 1996 by Bocquet to describe patients exhibiting a drug-induced condition characterized by an extensive rash, fever, lymphadenopathy, hematologic abnormalities, hepatitis, and involvement of the kidneys, lungs, heart, or pancreas [8]. In the review of 172 cases reported as DRESS or drug hypersensitivity syndrome in the literature by using the Regis CAR scoring system, carbamazepine remains the mostly reported (27\% of cases) [2]. But a variety of other drugs, such as allopurinol, minocycline, dapsone, sulfasalazine, mexiletine, anti-retrovirals and NSAIDs have also been associated with DRESS [3]. Regis CAR and Japanese consensus group have developed specific criteria for making the diagnosis of DRESS. To meet the definition of DRESS, patients must have three of the four main Regis CAR criteria: an acute rash, fever above $38^{\circ} \mathrm{C}$, lymphadenopathy at two sites, involvement of at least one internal organ, and abnormalities in lymphocyte and eosinophil counts. Additional criteria include hospitalization and that the reaction is suspected to be drug-related. Concerning Japanese consensus group the diagnosis requires meeting seven of the nine criteria in this system or all of the first five: a maculopapular rash developing $>3$ weeks after drug initiation, clinical symptoms continuing $>2$ weeks after stopping therapy, fever $>38^{\circ} \mathrm{C}$, liver abnormalities (ALT $>100 \mathrm{IU} / \mathrm{L}$ ) or other organ involvement, leukocytosis, atypical lymphocytes, eosinophilia, lymphadenopathy, or HHV-6 reactivation [7].

The reaction often starts with ever shortly followed by rash, which is usually pruritic, and variable degrees of lymphadenopathy. The cutaneous eruption consists of a morbilli form rash. Initially, the upper trunk, face, upper extremities are affected and followed by involvement of lower extremities. Swelling of the face, with marked periorbital involvement occurs in about $25 \%$ of patients. Tender lymphadenopathy can be seen in most patients (70---75\%) early in the illness, affecting predominantly cervical nodes or generalized [9].

Other features of DRESS include visceral involvement (hepatitis, pneumonitis, myocarditis, pericarditis, nephritis, pancreatitis, encephalitis, thyroiditis, colitis. The liver is reported to be the most frequently involved internal organ [10-12]. Hepatitis with isolated elevation of liver transaminases is common. A cholestatic injury pattern is seen in a minority of patients [13]. Elevations in 
liver enzymes usually continue to persist for several days after discontinuation of the offending drug. Liver failure, although rare, contributes to the leading causes of death $[10,14]$.

In 2010, the French Society of Dermatology published a consensus on the management of DRESS syndrome. According to this consensus, the absence of signs of severity, they recommend topical corticosteroids, emollients and H1-antihistamines. In the presence of signs of severity (transaminases greater than five times normal, renal impairment, pneumonia, haemophagocytosis or cardiac involvement), they propose corticosteroids equivalent to $1 \mathrm{mg} / \mathrm{kg}$ per day of prednisolone. If there are life- threatening signs: haemophagocytosis with bone marrow failure, encephalitis, severe hepatitis, renal failure, respiratory failure), they suggest steroids generally associated with intravenous immunoglobulin (IVIG) at a dose of $2 \mathrm{~g} / \mathrm{kg}$ over 5 days [15]. The mortality rate is reported to be between 52 and $20 \%$ and has been correlated with the degree of hepatic or renal involvement [16,17].

\section{Conclusion}

The diagnosis of DRESS should be highly suspected with the presence of skin rash, liver involvement, fever, hyper eosinophilia, and lymphadenopathy. The use of systemic corticosteroids is classically reported in case of organ- or life-threatening disease. Besides the prompt withdrawal of causative drug as standard of care, further studies are needed to recommend specific treatment guidelines.

\section{Conflicts of interest}

The authors have no conflicts of interest to declare.

\section{Right to Privacy and Informed Consent}

The authors have obtained the written informed consent of the patients or subjects mentioned in the article. The corresponding author is in possession of this document.

\section{References}

1. Choudhary S, McLeod M, Torchia D, Romanelli P (2013) Drug Reaction with Eosinophilia and Systemic Symptoms (DRESS) syndrome. J Clin Aesthet Dermatol 6(6): 31-37.
2. Cacoub P, Musette P, Descamps V, Meyer O, Speirs C, et al(2011) The DRESS syndrome: a literature review. Am J Med 124(7): 588-597.

3. EL omairi N, Abourazzak S, ChaoukiS, Atmani S, Hida M (2014) Drug Reaction with Eosinophilia and Systemic Symptom (DRESS) induced by carbamazepine: a case report and literature review. Pan Afr Med 18: 1-9.

4. Blanca M, Doña I, Torres M J, Campo P, Burgos JL, et al. (2009) Non steroids anti-inflammatory drugs (NSAIDs) intolerance versus allergy: patterns of response and drug involved. J Allergy Clin Immunol 123(3): S239.

5. Sanchez-Borges M, Capriles-Hulett A, CaballeroFonseca F (2002) NSAID-induced urticaria and angioedema: A reappraisal of its clinical management. Am J Clin Dermatol 3(9): 599-607.

6. Puijenbroek V, Egberts AC, Meyboom RH, Leufkens HG (2002) Different risks for NSAID-induced anaphylaxis. Ann Pharmacother 36(1) : 24-29.

7. van der KlauwW, Stricker B (1996) Drug-associated anaphylaxis: 20 years of reporting in The Netherlands (1974-1994) and review of the literature. Clin Exp Allergy 26(12): 1355-1363.

8. Bocquet H, Boagot M, Roujeau JC (1996) Druginduced pseudolymphoma and drug hypersensitivity (Drug rash with eosinophilia and systemic symptoms: DRESS). Semin Cutan Med Surg 15(4): 250-257.

9. Alkhateeb H, Said S, Cooper CJ, Gaur S, Porres-Aguilar M (2013) DRESS syndrome following ciprofloxacin exposure: an unusual association. Am J Case Rep 14: 526-528.

10. ShioharaT, InaokaM, KanoY (2006) Drug-induced hypersensitivity syndrome (DIHS): are action induced by a complex interplay among herpesviruses and anti-drug immune responses. Allergo lInt 55(1): 1-8.

11. Giri PP, Roy S, Bhattyacharya S, Pal P, Dhar S (2011) Dress syndrome with sepsis, acute respiratory distress syndrome and pneumomediastinum. Indian J Dermatol 56(6): 763-765.

12. Kim DH, KohYI (2014) Comparison of diagnostic criteria and determination of prognostic factors for drug reaction with eosinophilia and systemic 


\section{Advances in Clinical Toxicology}

symptoms syndrome. Allergy Asthma Immunol Res 6(3): 216-221.

13. Cardoso CS, Vieira AM, Oliveira AP (2011) DRESS syndrome: a case report and literature review. BMJ Case Rep 3: 2011.

14. Cornejo-Garcia JA, Blanca-López N, Doña I, Andreu I, Agúndez JA, et al. (2009) Hypersensitivity Reactions to Non-Steroidal Anti-Inflammatory Drugs. Current Drug Metabolism 10(9): 971-980.

15. Descamps V, Ben Saïd B, Sassolas B, Truchetet F, Avenel-AudraneM, et al. (2010) Prise en charge du drug reaction with eosinophilia and systemic symptoms (DRESS). Annales de dermatologie et de vénéréologie 137(11): 703-708.

16. Chen YC, Chiu HC, Chu CY (2010) Drug reaction with eosinophilia and systemic symptoms: a rétrospectives study of 60 cases. Arch Dermatol 146(12): 13731379.

17. Chiou CC, Yang LC, Hung SI, Chang YC, Kuo TT, et al. (2008) Clinicopathological features and prognosis of drug rash with eosinophilia and systemic symptoms: a study of 30 cases in Taiwan. J Eur Acad Dermatol Venereol 22(9): 1044-1049.

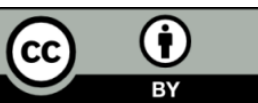

